\title{
Galectin-3 in the Gut-liver axis Regulates Autistic- like Behaviors by Mechanism Associated with Cerebral Shank-3+ cell Niches in BALB/c Mice
}

\section{Felipe Lemos}

Federal University of Rio de Janeiro

\section{Caio Prins}

Federal University of Rio de Janeiro

\section{Raul Carpi-Santos}

Federal University of Rio de Janeiro

Ingrid Waclawiak

Federal University of Rio de Janeiro

\section{Sofia Santos}

Universidade de São Paulo

\section{Emerson Bernardes}

Universidade de São Paulo

João Peça

University of Coimbra

\section{Ana Martinez}

Federal University of Rio de Janeiro

Cláudia Benjamim

Federal University of Rio de Janeiro

Roberto Luisetto

University of Padua

\section{Valéria Mello-Coelho}

Federal University of Rio de Janeiro

\section{Roger Chammas}

Universidade de São Paulo

Felipe Oliveira ( $\square$ felipe@histo.ufri.br)

Federal University of Rio de Janeiro

\section{Research Article}

Keywords: Galectin-3, gut, liver, brain, behavior, autism, oxidative stress, cow's milk, mice model of autism, shank-3 
Posted Date: January 14th, 2021

DOl: https://doi.org/10.21203/rs.3.rs-141155/v1

License: (c) (i) This work is licensed under a Creative Commons Attribution 4.0 International License. Read Full License 


\section{Abstract}

Galectin-3 stabilizes cell-cell junctions and regulates inflammatory pathways in the gut-liver axis. Galectin-3 knockout (Lgals3 ${ }^{-/}$) mice have atypical behaviors by obscure mechanisms. Given that BALB/c mice naturally develop low-sociability, stereotypies and restrict interest, they have been included as autism experimental model. Our major aims were to investigate whether galectin-3 in the gut-liver axis interferes with autistic-like behaviors analyzing BALB/c Lgals3 ${ }^{-/-}$mice or under partial inhibition of galectin-3 oral intake of cow's milk for 7 days. Behavioral patterns were assessed using a three-chambers test, open field, and self-grooming. Histological analysis and immunohistochemistry (Galectin-3, NOS-2, Iba-1, Ki-67, DII-4, Shank-3, Synaptophysin and Drebrin) were performed in gut, liver, and/or brain. $\mathrm{Lgals}^{-/-}$mice amplified stereotypies, social retraction and restrict interest associated with reduction of cerebral Shank $-3^{+}$cells. In Lgals $3^{+/+}$mice, cow's milk intake also amplified atypical behaviors, reduced galectin-3 in enterocytes and Kupffer cells, and disturbed niches of intestinal $\mathrm{KI}^{+} 7^{+}$and DII-4 $4^{+}$cells and hepatic NOS2 ${ }^{+}$cells. In the brain of milk-treated mice, Iba- $1^{+}$microglial cells and NOS2 ${ }^{+}$Purkinje cells were increased whereas Shank $-3^{+}$and Drebrin ${ }^{+}$Synaptophysin ${ }^{+}$cells were reduced suggesting, for the first time, that galectin-3 interferes with autistic behavior. Perhaps, a perspective to new therapies in genetically predisposed individuals to atypical behaviors.

\section{Introduction}

Autism spectrum disorder (ASD) is a neurodevelopmental condition characterized by atypical behaviors, including low sociability, restrict interests, stereotypies, and neuroinflammatory conditions ${ }^{1}$. The etiology and pathophysiology of ASD are poorly understood, although is consensus that causative factors are genetic and environmental ${ }^{2}$. The advent of experimental mice models has contributed to identify signaling pathways involved with ASD and associated comorbidity, such as gastrointestinal symptoms ${ }^{3}$.

The absence of reliable biomarker reinforces the necessity to explore experimental models in order to elucidate cellular and molecular mechanisms involved with ASD progression ${ }^{4}$. In this context, BALB/c and Shank-3 deficient (Shank- $3^{-/-}$) mice develop social, behavioral and neuroanatomical patterns compatible with ASD ${ }^{5,6}$. Shank-3 regulates synaptic functions in the CNS and Shank-3 $3^{-/-}$mice have severe synaptic dysregulation, cortico-striatal dysfunctions and gastrointestinal (GI) disorders correlated with ASD signals ${ }^{3,6}$.

Gl comorbidities are common in autistic individuals, frequently associated with intellectual disabilities and behavioral problems ${ }^{7}$. Pathologic intestinal permeability and reduced capacity of hepatic sulfation have been recurrent in these patients ${ }^{8}$, indicating that gut-liver axis can be a therapeutic target to improve GI comorbidities and behavioral-related symptoms. In this context, diets that interfere with gut-liver functions have potential to assuage or exacerbate symptoms in ASD patients with genetic predisposition 9 . 
In order to investigate potential gut-liver markers involving extracerebral factors and ASD symptoms, galectin-3 emerges as molecular target. Galectin-3, a $\beta$-galactoside binding protein that controls cell-cell and cell-extracellular matrix interactions in distinct tissues ${ }^{10}$, is synthesized by enterocytes, stabilizing desmoglein-2 in desmosomal junctions ${ }^{11}$. Hepatic galectin-3 is produced by Kupffer cells and cholangiocytes but is absent in health hepatocytes ${ }^{12,13}$.

Galectin-3 knockout (Lgals3 ${ }^{-/-}$) mice have markedly gut-liver axis disorders, including severe disorganization of intestinal epithelial cells and hepatic progenitor cell niches ${ }^{14,15}$. Recently, it was demonstrated that $\mathrm{Lgals}^{-/-}$mice have atypical behavior in home cage movement ${ }^{16}$ and impaired emotional response to stress associated with obsessive-compulsive-like disorder ${ }^{17}$. However, galectin-3 is absent in healthy brain of mice ${ }^{18}$. Based on these data, we hypothesize that disturbances on gut-liver axis detected in $\mathrm{Lgals}^{-/-}$mice could be associated with atypical behavioral pattern. Here, for the first time, intestinal and hepatic galectin-3 can be included as experimental target to alleviate comorbidities associated with autistic-like behavioral parameters.

\section{Results}

\section{Absence of galectin-3 disturbed behavioral patterns in BALB/c mice}

To investigate a possible interference of galectin-3 in ASD-associated phenotypes, BALB/c Lgals ${ }^{+/+}$and $\mathrm{Lgals}^{-/-}$mice were submitted to behavioral tests. Repetitive stereotyped behavior was severely increased in Lgals3 $3^{-/-}$mice. These mice expended approximately $20 \%$ of time performing stereotyped movements whereas controls used only $5 \%$ of experimental time with this behavior (Fig. 1A). In the absence of galectin-3, mice showed limited interest for new object, expending about $25 \%$ of time exploring a new object. In contrast, control Lgals $3^{+/+}$mice demonstrated higher interest for new object than $\mathrm{Lgals}^{-/-}$mice, using $45 \%$ of time exploring a toy (Fig. 1B), indicating that mice lacking galectin-3 have restrict interest.

Parameters of sociability were also evaluated in both mice groups, but they were partially affected by the absence of galectin-3. Social retraction was significantly increased in Lgals $3^{-/-}$mice and the percentage of time in isolation and/or non-interacting changed from approximately $5 \%$ in controls to around $15 \%$ of total time in Lgals $3^{-/-}$mice (Fig. 1C). On the other hand, both mice groups spent similar percentage of total time seeking social contact with unfamiliar mouse (Fig. 1D). The three chambered test revealed that $\mathrm{BALB} / \mathrm{C} \mathrm{Lgals} 3^{+/+}$mice have more interest for toys in detriment to familiar mouse. Instead, Lgals $3^{-/-}$ mice spent less time on chamber with toy and more time in chamber with familiar mouse (Fig. 1E). Moreover, this test revealed that social contact with familiar mouse was significantly increased in the absence of galectin-3 when compared with control mice (Fig. 1E). 
The increased repetitive behavior associated with partial changes in sociability in the absence of galectin3 indicated possible imbalance on Shank-3 pathways, since Shank-3 is a scaffolding protein that stabilizes glutamatergic receptors in $\mathrm{CNS}^{19}$. In the brain of control mice, Shank-3 was widely distributed throughout the cortical regions mostly expressed by neurons and glial cells (Fig. 1F). In contrast, Lgals3-/ - mice showed reduced proportion of Shank $-3^{+}$cells (Fig. 1G). Approximately $80 \%$ of cortico-cerebral cells were positive to Shank-3 in control mice while only $15 \%$ of these cells were marked to Shank-3 in Lgals ${ }^{-1}$ - mice (Fig. 1H). Together, these data suggest that the reduction of Shank- $3^{+}$cells in the absence of galectin-3 could be associated with important behavioral disturbances, some of them related to ASD.

\section{Cow's milk supplementation reduces galectin-3 in the gut- liver axis of $B A L B / c$ mice}

Given that $\mathrm{Lgals}^{-/-}$BALB/C mice showed atypical behaviors, $\mathrm{Lgals}^{+/+} \mathrm{BALB} / \mathrm{C}$ mice were supplemented with cow's milk containing lactose and other glycans that inhibit galectin-3 functions. On day 7 of treatment, the gut of control mice was hallmarked by enterocytes strongly positive to galectin-3 mainly in the tip of villi (Fig. 2A). In contrast, enterocytes of milk-supplemented mice were rarely positive to galectin3 (Fig. 2B). The percentage of galectin- $3^{+}$cells was significantly reduced in the epithelial tissue in milksupplemented mice when compared with control group (Fig. 2C). However, in the lamina propria, the percentage of galectin- $3^{+}$cells was similar between both groups of mice (Fig. 2D). These data indicate that milk daily diet significantly reduced the levels of galectin-3 in the intestinal epithelial cells of mice.

Milk intake also interfered with hepatic galectin- $3^{+}$cells. In controls, galectin-3 was preferentially found in sinusoidal cells with morphology compatible with Kupffer cells. It is important to note that majority, but not all Kupffer cells were positive to galectin-3 in these mice (Fig. 2E). In milk-treated mice, Kupffer cells were poorly stained to galectin-3 and majority of Kupffer cells was negative to galectin-3 (Fig. 2F). The percentage of hepatic galectin- $3^{+}$cells was significantly reduced in milk-supplemented mice when compared with control group (Fig. 2G). Indeed, the percentage of Kupffer cells expressing galectin-3 was significantly reduced after milk treatment (Fig. $2 \mathrm{H}$ ). It is important to note that galectin- $3^{+}$cells were not detected in the cerebral cortex and cerebellum on day 7 after milk treatment (data not shown). It is plausible to attribute the reduction of galectin-3 expressing cells in the gut-liver axis with the intake of milk.

\section{Cow's milk affects histological niches in the gut and liver of BALB/c mice}

In order to investigate whether reduction of galectin-3 associated with cow's milk intake affects the histological architecture of gut-liver axis, samples of small intestine and liver were analyzed on day 7 of supplementation. Apparently, general villi organization was not affected by milk consumption (Supplementary Fig. 1A and 1B). However, cryptal $\mathrm{KI}^{+} 7^{+}$cell distribution was significantly disorganized in mice after milk consumption when compared with control group (Fig. 3A and 3B). Moreover, the reduction of galectin-3 in enterocytes and atypical organization of $\mathrm{KI}^{+} 7^{+}$cells were correlated with higher 
concentration of DLL-4 $4^{+}$cells. In the gut mucosa, these DLL- $4^{+}$cells were frequently detected on basal regions of the crypts and lamina propria in controls (Fig. 3C). On the other hand, DLL-4 ${ }^{+}$cells were widely distributed throughout the crypts and more frequent in the lamina propria of milk supplemented mice (Fig. 3D).

In the liver, the effects of milk consumption were observed in the cytoplasm of hepatocytes. These cells showed significant perinuclear vacuolation when preferentially localized in the acinar zone 1 , surrounding the portal triads (Supplementary Fig. 1C and 1D). Vacuoles in hepatocytes are indicative of glycogen or lipid deposits. Using PAS staining, it was found that liver of milk-supplemented mice showed significant abnormal glycogen accumulation when compared with control group (Fig. 3E and 3F). To associate this parameter with increased susceptibility to hepatic injury, $\mathrm{NOS}^{+}$cells were evaluated, and it was observed a robust number of $\mathrm{NOS}^{+}$cells in the liver of milk- supplemented mice, compared to control mice (Fig. 3G-H). Clearly, the consumption of cow's milk affected the homeostasis of intestinal and hepatic cells.

\section{Cow's milk increased cerebellar IBA-1 + microglial cells and NOS-2 + Purkinje cells}

To investigate whether gut-brain axis was affected by cow's milk consumption, SNC samples were analyzed and associated with reduction of galectin- $3^{+}$cells in the small intestine and liver. Cow's milk intake did not affect histological parameters in the cerebellum (Supplementary Fig. 2A and 2B) and cerebellar galectin- $3^{+}$cells were not detected in control or milk-supplemented mice (data not shown). However, the percentage of IBA- $1^{+}$microglial cells increased after milk consumption. These cells represented approximately $4 \%$ of total cerebellar cells in controls and $10 \%$ in milk-treated mice (Fig. 4AC).

The possible induction of oxidative stress in the liver after milk consumption was critical to suggest systemic signals. Thus, NOS2 ${ }^{+}$cells were investigated in the cerebellum of these mice. In fact, milk consumption was associated with significant increase of percentage of Purkinje cells expressing NOS2 ${ }^{+}$. These cells represented approximately $30 \%$ of total cerebellar cells in controls whereas the number increased to $65 \%$ in cow's milk supplemented mice (Fig. 4D-F). Together, these data indicate that bovine milk intake affects microglial and Purkinje cell niches in the cerebellum of mice. At least in part, these findings seem to be associated with atypical distribution of galectin-3 in gut-liver axis.

\section{Cow's milk disturbed microglial cell niches and synapses in cortico-cerebral regions}

Consistent with cerebellum, the numbers of IBA- $1^{+}$cells were significantly increased in cerebral cortex of mice supplemented with cow's milk (Fig. 5A and 5B). In controls, cortico-cerebral IBA-1 ${ }^{+}$cells represented approximately $5 \%$ of total cells whereas in milk-treated mice this percentage increased to $9 \%$ (Fig. $5 \mathrm{C}$ ). 
Again, milk intake also did not interfere with histological architecture (Suppl. Figure 2C and 2D) and no cells expressing galectin-3 were found (data not shown).

The organization of synapses were also investigated in mice supplemented with bovine milk. In the cortico-cerebral regions of control mice, synaptophysin ${ }^{+}$and drebrin ${ }^{+}$cells were widely dispersed throughout the brain parenchyma, frequently colocalized in the tissue (Fig. 5D). On the other hand, mice supplemented with cow's milk revealed similar levels of synatoptophysin staining, but significant reduction of drebrin immunoreactivity. Co-localization images corroborated with drastic reduction of synaptic puncta (Fig. 5E). Using controls as $100 \%$ fluorescence intensity, it was possible to identify that synaptophysin reactivity was apparently, but not statistically, reduced in the cortex of milk-supplemented mice in comparison with controls. In contrast, the intensity of drebrin decreased near of $90 \%$ in mice after milk consumption. Therefore, the levels of formed synapses, as seen by the colocalization of pre (synaptophysin) and postsynaptic (drebrin) showed similar percentual reduction (Fig. 5F).

\section{Lack of galectin-3 was correlated with behavioral changes in mice supplemented with cow's milk}

Given that $\mathrm{Lgals}^{-/-}$BALB/c mice showed atypical behavioral pattern and $\mathrm{Lgals} 3^{+/+} \mathrm{BALB} / \mathrm{c}$ mice reduced the levels of galectin-3 in the gut-liver axis after consumption of milk, it was plausible to investigate a possible correlation between these findings. Consistently, shank- $3^{+}$cells were widely distributed in the cerebral cortex of control mice (Fig. 6A). As observed in Lgals ${ }^{-/-}$mice (Fig. 1), mice supplemented with cow's milk showed an intermediate pattern of Shank-3 $3^{+}$cell distribution (Fig. 6B). Furthermore, quantitative analysis revealed that near of $80 \%$ of cells in control mice were shank- $3^{+}$. In contrast, the percentage significantly decreased to approximately $40 \%$ of cortical cells in mice supplemented with milk (Fig. 6C).

To correlate low levels of galectin-3 in the gut-liver and behavioral changes in Lgals $3^{-/-}$mice, milk-treated mice were submitted to behavioral tests. After milk consumption, stereotypies were significantly amplified in Lgals $3^{+/+}$mice (Fig. 6D). These mice also demonstrated restrict interest for new object after milk consumption when compared with control mice (Fig. 6E). Three chambered tests revealed that milksupplemented mice spend more time with toys than other (familiar) mouse (Fig. 6F). Together, these data linked behavioral changes detected in the absence of galectin-3 with disturbances in the gut-liver axis hallmarked by reduction of galectin-3 in gut-liver axis.

\section{Discussion}

For the first time, it is suggested that intestinal and hepatic galectin- $3^{+}$cells interfere with autistic-like behaviors. Total (Lgals3 ${ }^{-/-}$mice) or partial (Lgals $3^{+/+}$mice supplemented with cow's milk) absence of galectin-3 in the gut-liver axis amplified stereotypies, restrict interest and social retraction in BALB/c mice. These behavioral phenotypes were related with disturbed niches of intestinal epithelial cells, hepatocytes and Kupffer cells in the liver, and shank- $3^{+}, \mathrm{Iba}_{-1}{ }^{+}, \mathrm{NOS}^{+}$and drebrin ${ }^{+}$cells in the CNS. 
BALB/c mice showed significant low sociability, self-aggressive behaviors, and stereotypies when compared with neurotypical C57BL/6 mice ${ }^{5}$. Our data revealed that both total absence (Lgals3 ${ }^{-/-}$ $\mathrm{BALB} / \mathrm{c}$ mice) or reduction of galectin-3 in the gut-liver axis induced by milk supplementation increased atypical behaviors. The role of galectin-3 as immunomodulatory factor in the gut and liver has been attracted the attention of specialists ${ }^{15,20}$.

Galectin-3 colocalizes with desmoplakin-1 and desmoglein-2 contributing to selective permeability in enterocytes ${ }^{21}$. In Lgals $3^{-/-}$mice, enterocytes have ultrastructural defects in cytoskeleton and basolateral membrane domains resulting in aberrant traffic of luminal molecules ${ }^{14}$. In the liver, galectin-3 plays hepatoprotective functions in distinct conditions ${ }^{12,22,23}$. Lgals3 $^{-/-}$mice develop severe liver steatosis in diet models ${ }^{24}$, NASH in aging ${ }^{25}$, low regenerative capacity ${ }^{15}$, and abnormal organization of collagen fibers in experimental fibrosis ${ }^{26,27}$. Maybe, the reduction or total absence of galectin-3 could modify the intestinal permeability allowing the circulation of luminal components with potential to affect CNS functions.

The strategy to reduce galectin-3 in the gut-liver axis of wild type mice seemed efficient, given that mice supplemented with cow's milk reduced the epithelial levels of galectin-3 in the intestinal villus and hepatic Kupffer cells. Cow's milk carries galectin-3 inhibitors, including lactose ${ }^{28}$. Intriguingly, milk consumption caused a significant imbalance in intestinal $\mathrm{KI}^{+}$and $\mathrm{DII}-4^{+}$cell niches, indicating an abnormal turnover of epithelial cells. In corroboration, $\mathrm{KI} 67^{+}$cell niches are disorganized during intervention with milk oligosaccharides and epithelial barrier dysfunctions that permit the abnormal flux of microbiota compounds ${ }^{29-32}$. DII-4 normally synthesized by intestinal epithelial cells is significantly increased during mucosa inflammation ${ }^{33,34}$. In addition, DII-4/- mice showed reduced niches of $\mathrm{KI}^{-1} 7^{+}$cells in the gut ${ }^{35}$.

In the liver, milk supplementation was linked to abnormal glycogen accumulation in hepatocytes. Although glycogen naturally accumulates after milk consumption within $18 \mathrm{~h}$ of last supplementation ${ }^{36}$, long-time glycogen deposit and hydropic degeneration are compatible with subacute liver injury ${ }^{37,38}$. Regarding to increased number of NOS2 ${ }^{+}$cells, this phenotype has been associated with oxidative stress induced by chemical hepatotoxic processes ${ }^{39-41}$.

The atypical pattern of behavior in the absence or partial reduction of galectin-3 in the gut-liver axis led us to investigate the distribution of shank $-3^{+}$and $\mathrm{Iba}-1^{+}$cells, possible oxidative stress sites and the organization of some synaptic proteins in the CNS. Shank-3 stabilizes glutamatergic receptors in synapses ${ }^{42}$ and shank-3 $3^{-/-}$mice exhibited neurodevelopmental disturbances in neurons of prefrontal cortex ${ }^{43}$, GABAergic interneuron dysfunctions ${ }^{44}$, and social deficits followed by repetitive stereotyped behaviors ${ }^{45,46}$. In our experimental conditions, reduction or lack of galectin-3 was concomitant with decreased numbers of shank $-3^{+}$cell in the cerebral cortex, suggesting that gut-liver axis disorders and atypical behaviors can be correlated by, at least in part, cellular signaling pathways involving galectin-3 and shank-3. 
The cerebral cortex of mice marked by partial inhibition of galectin-3 in the gut-liver axis was also characterized by increased numbers of IBA- $1^{+}$microglial cells. The same pattern was observed in the cerebellum of these milk-supplemented mice. It is possible that extracerebral inflammation, including inflammatory bowel diseases, activates microglial cells in the CNS ${ }^{4-49}$. Particularly in ASD, gliosis and neuroinflammation events have been described in patients and murine models ${ }^{50,51}$. The cerebellum of BTBR T + tf/J (BTBR) mice, an relevant experimental model to study ASD ${ }^{52,53}$, has increased numbers of $\mathrm{Iba}-1^{+}$cells and NOS2 expression correlated with stereotypies ${ }^{54,55}$. Our data corroborated with these authors since the cerebellum of milk-supplemented mice was also characterized by increased numbers of Iba-1 + microglial cells and NOS2 + Purkinje cells. These data incite a hypothesis that early damages outside of the CNS can be a trigger to initiate behavioral changes.

Pre and postsynaptic proteins were also investigated in mice supplemented with cow's milk. Drebrin ${ }^{+}$ cells were significantly reduced in the cerebral cortex of mice receiving milk. Drebrin is an actin-binding protein present in postsynaptic glutamatergic neurons whose concentration in dendritic spines organizes cytoskeleton and morphology of the cells. Dysfunctions were associated with Alzheimer's disease, schizophrenia and ASD related to post synaptic accumulation of glutamatergic receptors ${ }^{56,57}$. The reduction of shank $-3^{+}$and drebrin ${ }^{+}$cells in the CNS can be directly associated with behavioral changes observed after milk consumption. The distribution of galectin- $3^{+}$cells in the gut-liver axis and cortical shank- $3^{+}$and drebrin ${ }^{+}$cells could be monitored in other ASD experimental models. Perhaps, some genetic predisposition is required to establish this phenotype and more detailed studies are necessary.

In our scenario, we suggest that galectin-3 may be related to the onset of ASD symptoms as co-factor together environmental and genetic causes still for the most part unknown. Coherently with our observation in this study, we can hypothesize that the role of galectin-3 could be crucial at different levels. At early stages, during neurodevelopment and subsequently regulating the synaptic plasticity network pruning and maintenance not directly but through several mediators as BNDF, GABA-AR, NGF, Neurtrophin-3, Synaphtophysin A and Drebrin ${ }^{58,59}$. At peripheral level, this study confirms the key role of galectin-3 as cross-link factor between innate immunity, visceral sensitivity and gut tissues development and homeostasis. The delicate balance between these functions and the influence of dietary habits should be accurately investigated in order to define every molecular pathway involved in the onset of behavioral, cognitive and neurological disturbances associated with autism.

In conclusion, $\mathrm{Lgals}^{-/-}$mice and $\mathrm{Lgals} 3^{+/+}$mice supplemented with cow's milk showed atypical behaviors compatible with autistic-like symptoms. Considering that BALB/c Lgals $3^{+/+}$mice have been used as experimental model of ASD, for the first time, it is suggested that galectin-3 interferes with gastrointestinal comorbidities and autistic behavior. Data revealed that absence or reduction of galectin-3 in the gut-liver axis increased stereotyped movements, affected sociability indexes and amplified restrict interest for objects. However, it is important to reinforce that cow's milk did not cause ASD. Although galectin- $3^{+}$cells were not detected in CNS of both mice groups, it has been suggested that galectin-3 in the gut-liver axis interferes with brain functions associated with ASD symptoms in genetically 
predisposed mice. These findings pointed to mechanisms outside of the CNS can be studied as therapeutic target to alleviate symptoms of gastrointestinal comorbidities associated with autism.

\section{Methods}

\section{Animals and milk supplementation}

Six-week-old male BALB/c Lgals3 ${ }^{+/+}$and Lgals3 $^{-/-}$mice were obtained from the colony bred at the Federal University of Rio de Janeiro, Brazil. The experimental protocols were approved by local Animal Ethics Committee of Federal University of Rio de Janeiro, Brazil (protocol number: 071/19) in accordance with the guidelines provided the Brazilian College of Animal Experimentation. Mice were randomly separated in four groups: (I) Lgals $3^{+/+}$and (II) $\mathrm{Lgals}^{-/-}$mice without any supplementation, (III) $\mathrm{Lgals}^{+/+}$ mice supplemented with $200 \mu \mathrm{L}$ three times a day with commercial cow's milk for 1 week by oral gavage, and (IV) $\mathrm{Lgals}^{+/+}$mice receiving $200 \mu \mathrm{L}$ of water by gavage at the same volume and time (control of group III). This study was carried out in compliance with the ARRIVE guidelines.

\section{Histological analysis and immunohistochemistry}

Gut, liver and brain of mice were collected and immediately fixed in $4 \%$ buffered formalin for 72 hours at room temperature. Brain was segmented into cortex and cerebellum fragments. Histological samples were dehydrated, diaphanized, individually embedded in paraffin, sectioned into $3 \mu \mathrm{m}$ thick slices, and stained with hematoxylin and eosin dyes ${ }^{22}$. To immunohistochemistry, sections were heated in Trilogy ${ }^{\text {TM }}$ (Sigma-Aldrich, USA). Peroxidase and nonspecific antibody interactions were inhibited by $3 \% \mathrm{H}_{2} \mathrm{O}_{2}$ and BSA $8 \%$ diluted in $0.002 \%$ PBS-Tween 20. Primary antibodies: anti-galectin-3 (clone M3/38, Cell Bank of Rio de Janeiro, Brazil), anti-NOS2, anti-IBA-1 (BD Bioscience, USA), anti-KI67 (Vector, UK) and antiSHANK3 (Sigma-Aldrich, USA). After secondary antibody incubation, samples were treated with streptavidin - peroxidase (Sigma-Aldrich, USA), diaminobenzidine (DAB) used as substrate, and samples counterstained with Harris' hematoxylin. Percentage of positive cells was defined as number of marked cells compared to total cells (500 cells per sample). Morphological analysis was performed using highpower microscopy (Zeiss-Axioplan, Germany) and images were acquired by Evolution MP 5.0 RTV-Color camera (Media Cybernetics, Canada).

\section{Immunofluorescence}

Paraffin sections were deparaffinize with 2 washes with xylene for 15 minutes each. After deparaffinization, the sections were submitted to a gradient of ethanol solution (100-50\%) of 2 minutes each. Then, samples were incubated with PBS 1 x/0.05\% tween 20 for 30 minutes. After that, the sections were washed with PBS $1 \times / 0.05 \%$ tween 20 , incubated in citrate buffer at $98^{\circ} \mathrm{C}$ for 40 minutes and treated with blocking buffer ( $3 \%$ bovine serum albumin, $5 \%$ normal goat serum [Sigma-Aldrich, USA] diluted in PBS 1X/0.05\% tween 20). After blocking, the samples were incubated with the primary antibodies overnight, washed 3 times with PBS 1X/0.05\% tween 20 and incubated with secondary antibodies. Nuclei were counterstained with DAPI (Sigma-Aldrich, USA). Slices were mounted with DAKO Mounting Media 
and imaged on a confocal microscope (Leica TCS SPE). Primary antibodies: anti-NOS2 (BD Bioscience, USA), anti-synaptophysin and Drebrin (Milipore, USA). Secondary antibodies: Anti-rat IgG Alexa 488 (BD Bioscience, USA), anti-Mouse 594 for synaptophysin and Anti-Rb 488 for drebrin (Molecular Probes, USA).

After capturing 6-8 images per group of cortical external granular layer, the green and red channels were aligned, and the colocalized puncta number was quantified using the Puncta Analyzer plug-in in NIH ImageJ as previously described ${ }^{60}$. Experiments were done in duplicate.

\section{Behavioral assays}

Prior to behavior tests, mice were acclimated to the testing rooms and platforms over five consecutive days. After 1 hour in the sound-isolated room test, each mouse was individually adapted inside the testing platforms. To stereotyped motor behavior, each mouse was individually recorded for 15 minutes, 5 frames/second, from a webcam positioned $60 \mathrm{~cm}$ in front of a translucent acrylic cage with $10 \mathrm{~cm} \mathrm{x}$ $10 \mathrm{~cm} \times 15 \mathrm{~cm}$ to monitor stereotyped motor attitudes such as paw flapping, persistent self-grooming, circling and jumping. The time of each persistent stereotyped motor behavior and summed all time spent on these over the total filmed time were quantified in separate data. To sociability essays, each mouse was acclimated for 10 minutes in the three chambered test platform without novel colored cubic toy and same cage mouse, or 15 minutes inside the box with same cage animals. Each group of mice at a time were placed inside an open $90 \mathrm{~cm} \times 45 \mathrm{~cm} \times 7 \mathrm{~cm}$ box filled with nesting material and they were recorded, 5 frames/second for 15 minutes, from a webcam situated $60 \mathrm{~cm}$ above the box. Attitudes of social interaction and social play such as anal sniffing, nose sniffing, pouncing and pinning were recorded for each animal, and the seconds spent on these behaviors were quantified over the total filmed time. Social retraction which means the condition of non-interaction plus isolation from group of a vigil and not moving mouse was also counted and the percentage of time over the total recorded time was likewise quantified. To assess cognition in terms of general sociability and interest in social or spatial novelty was performed using one testing mouse which would be presented at the same time to (1) a cage containing a mouse already recognized by the testing mouse which is native from the same cage and from the same group; and (2) a cage containing a never presented before colored cubic toy. To check the sociability and social-spatial interest time, we counted the time spent by the testing mouse near the cage with the already known same cage mice or with the never presented colored cubic toy (near understood as $5.0 \mathrm{~cm}$ from the testing mouse nose tip pointing to one of these cages edge direction) or the time spent by the testing mouse touching, sniffing and exploring one of these cages. This experiment was recorded for 10 minutes by a webcam, 5 frames/second, positioned $1.5 \mathrm{~m}$ above the cage, and we quantified the exploration time over the total recorded time.

\section{Statistical analysis}

The statistical tests were accomplished using t-test, and significance threshold was fixed for $a=0.05$. Therefore, $P$ values $\leq 0.05$ were considered statistical differences. Each experiment was performed using 3-5 mice per group in three independent assays.

\section{Declarations}




\section{Acknowledgements}

We thank the Morphological Sciences Program (PCM), Federal University of Rio de Janeiro (UFRJ), where F.S.L. (CNPq scholarship) is coursing the Doctoral stage. CNPq: National Council for Scientific and Technological Development, Brazil.

\section{Author Contributions}

F.S.L., C.A.P., R.C., F.L.O.: designed experiments; F.S.L., C.A.P., R.C.S., I.W., S.N.S., F.L.O.: conducted the experiments; F.S.L., C.A.P., R.C.S, E.S.B., J.P., A.M.B.M., C.F.B., R.L., V.M.C., R.C., F.L.O.: analyzed the data; F.S.L, R.L., F.L.O.: wrote the main text. All authors reviewed the manuscript.

\section{Competing interest}

The authors declare no competing interests.

\section{References}

1. S. M. Matta, E. L. Hill-Yardin, P. J. Crack, The influence of neuroinflammation in Autism Spectrum Disorder. Brain Behav Immun, (2019).

2. A. Modabbernia, E. Velthorst, A. Reichenberg, Environmental risk factors for autism: an evidencebased review of systematic reviews and meta-analyses. Mol Autism 8, 13 (2017).

3. A. K. Sauer, J. Bockmann, K. Steinestel, T. M. Boeckers, A. M. Grabrucker, Altered Intestinal Morphology and Microbiota Composition in the Autism Spectrum Disorders Associated SHANK3 Mouse Model. Int J Mol Sci 20, (2019).

4. L. Shen et al., Biomarkers in autism spectrum disorders: Current progress. Clin Chim Acta 502, 41-54 (2020).

5. E. S. Brodkin, BALB/c mice: low sociability and other phenotypes that may be relevant to autism. Behav Brain Res 176, 53-65 (2007).

6. J. Peça et al., Shank3 mutant mice display autistic-like behaviours and striatal dysfunction. Nature 472, 437-442 (2011).

7. M. J. Penzol et al., Functional Gastrointestinal Disease in Autism Spectrum Disorder: A Retrospective Descriptive Study in a Clinical Sample. Front Psychiatry 10, 179 (2019).

8. K. Horvath, J. A. Perman, Autism and gastrointestinal symptoms. Curr Gastroenterol Rep 4, 251-258 (2002).

9. H. Cekici, N. Sanlier, Current nutritional approaches in managing autism spectrum disorder: A review. Nutr Neurosci 22, 145-155 (2019). 
10. S. Sciacchitano et al., Galectin-3: One Molecule for an Alphabet of Diseases, from A to Z. Int J Mol Sci 19, (2018).

11. K. Jiang et al., Galectin-3 regulates desmoglein-2 and intestinal epithelial intercellular adhesion. $J$ Biol Chem 289, 10510-10517 (2014).

12. F. L. de Oliveira et al., The Number of Liver Galectin-3 Positive Cells Is Dually Correlated with NAFLD Severity in Children. Int J Mol Sci 20, (2019).

13. M. Butscheid, P. Hauptvogel, P. Fritz, U. Klotz, D. M. Alscher, Hepatic expression of galectin-3 and receptor for advanced glycation end products in patients with liver disease. J Clin Patho/ 60, 415-418 (2007).

14. D. Delacour et al., Loss of galectin-3 impairs membrane polarisation of mouse enterocytes in vivo. $J$ Cell Sci 121, 458-465 (2008).

15. W. C. Hsieh et al., Galectin-3 regulates hepatic progenitor cell expansion during liver injury. Gut, (2014).

16. T. R. Chaudoin, S. J. Bonasera, Mice lacking galectin-3 (Lgals3) function have decreased home cage movement. BMC Neurosci 19, 27 (2018).

17. A. G. Sartim, M. A. Sartim, R. D. Cummings, M. Dias-Baruffi, S. R. Joca, Impaired emotional response to stress in mice lacking galectin-1 or galectin-3. Physiol Behav 220, 112862 (2020).

18. J. Liu, S. Huang, F. Lu, Galectin-3 and Galectin-9 May Differently Regulate the Expressions of Microglial M1/M2 Markers and T Helper 1/Th2 Cytokines in the Brains of Genetically Susceptible C57BL/ 6 and Resistant BALB/c Mice Following Peroral Infection With Toxoplasma gondii. Front Immuno/ 9, 1648 (2018).

19. A. L. Bey et al., Brain region-specific disruption of Shank3 in mice reveals a dissociation for cortical and striatal circuits in autism-related behaviors. Translational Psychiatry 8, 94 (2018).

20. V. Volarevic et al., Galectin-3 Regulates Indoleamine-2,3-dioxygenase-Dependent Cross-Talk between Colon-Infiltrating Dendritic Cells and T Regulatory Cells and May Represent a Valuable Biomarker for Monitoring the Progression of Ulcerative Colitis. Cells 8, (2019).

21. A. Gross et al., Desmoglein 2, but not desmocollin 2, protects intestinal epithelia from injury. Mucosal Immunol, (2018).

22. F. S. Lemos et al., Galectin-3 orchestrates the histology of mesentery and protects liver during lupuslike syndrome induced by pristane. Sci Rep 9, 14620 (2019).

23. R. Lukic et al., Potential Hepatoprotective Role of Galectin-3 during HCV Infection in End-Stage Renal Disease Patients. Dis Markers 2017, 6275987 (2017).

24. K. Nomoto et al., Disrupted galectin-3 causes non-alcoholic fatty liver disease in male mice. $J$ Pathol 210, 469-477 (2006).

25. Y. Nakanishi et al., Nonalcoholic steatohepatitis and hepatocellular carcinoma in galectin-3 knockout mice. Hepatol Res 38, 1241-1251 (2008).

26. F. L. Oliveira et al., in J Leukoc Biol. (United States, 2007), vol. 82, pp. 300-310. 
27. F. L. de Oliveira et al., Galectin-3, histone deacetylases, and Hedgehog signaling: Possible convergent targets in schistosomiasis-induced liver fibrosis. PLoS Negl Trop Dis 11, e0005137 (2017).

28. A. J. Noll et al., Galectins are human milk glycan receptors. Glycobiology 26, 655-669 (2016).

29. E. G. D. Hopkins, T. I. Roumeliotis, C. Mullineaux-Sanders, J. S. Choudhary, G. Frankel, Intestinal Epithelial Cells and the Microbiome Undergo Swift Reprogramming at the Inception of Colonic Citrobacter rodentium Infection. mBio 10, (2019).

30. Y. Wang et al., Fucosylation Deficiency in Mice Leads to Colitis and Adenocarcinoma. Gastroenterology 152, 193-205.e110 (2017).

31. C. Wang et al., Human Milk Oligosaccharides Protect against Necrotizing Enterocolitis by Inhibiting Intestinal Damage via Increasing the Proliferation of Crypt Cells. Mol Nutr Food Res 63, e1900262 (2019).

32. C. Yang et al., Molecular Mechanisms Underlying How Sialyllactose Intervention Promotes Intestinal Maturity by Upregulating GDNF Through a CREB-Dependent Pathway in Neonatal Piglets. Mol Neurobio/ 56, 7994-8007 (2019).

33. H. Shimizu et al., Distinct expression patterns of Notch ligands, DII1 and DII4, in normal and inflamed mice intestine. PeerJ 2, e370 (2014).

34. H. Imaeda et al., Interleukin-33 suppresses Notch ligand expression and prevents goblet cell depletion in dextran sulfate sodium-induced colitis. Int J Mol Med 28, 573-578 (2011).

35. T. Nakata et al., Data showing proliferation and differentiation of intestinal epithelial cells under targeted depletion of Notch ligands in mouse intestine. Data Brief 10, 551-556 (2017).

36. E.-S. F. Hammad, J. S. Striffler, R. R. Cardell, Morphological and biochemical observations on hepatic glycogen metabolism in mice on a controlled feeding schedule. Digestive Diseases and Sciences 27, 680-691 (1982).

37. N. C. Nayak et al., The nature and significance of liver cell vacuolation following hepatocellular injury-an analysis based on observations on rats rendered tolerant to hepatotoxic damage. Virchows Arch 428, 353-365 (1996).

38. E. Ulusoy, B. Eren, Histological Changes on Liver Glycogen Storage in Mice (Mus musculus) Caused by Unbalanced Diets. Clin Med Pathol 1, 69-75 (2008).

39. M. Kaibori, T. Okumura, K. Sato, M. Nishizawa, M. Kon, Inducible Nitric Oxide Synthase Expression in Liver Injury: Liver Protective Effects on Primary Rat Hepatocytes. Inflamm Allergy Drug Targets 14, 77-83 (2015).

40. B. H. Zhou et al., Fluoride-induced oxidative stress is involved in the morphological damage and dysfunction of liver in female mice. Chemosphere 139, 504-511 (2015).

41. T. Kumamoto et al., Role of nitric oxide synthesized by nitric oxide synthase 2 in liver regeneration. Liver Int 28, 865-877 (2008).

42. P. Monteiro, G. Feng, SHANK proteins: roles at the synapse and in autism spectrum disorder. Nat Rev Neurosci 18, 147-157 (2017). 
43. E. C. Cope et al., Immature Neurons and Radial Glia, But Not Astrocytes or Microglia, Are Altered in Adult Cntnap2 and Shank3 Mice, Models of Autism. eNeuro 3, (2016).

44. Q. Chen et al., Dysfunction of cortical GABAergic neurons leads to sensory hyper-reactivity in a Shank3 mouse model of ASD. Nat Neurosci 23, 520-532 (2020).

45. L. J. Duffney et al., Autism-like Deficits in Shank3-Deficient Mice Are Rescued by Targeting Actin Regulators. Cell Rep 11, 1400-1413 (2015).

46. L. Qin et al., Social deficits in Shank3-deficient mouse models of autism are rescued by histone deacetylase (HDAC) inhibition. Nat Neurosci 21, 564-575 (2018).

47. I. C. Hoogland, C. Houbolt, D. J. van Westerloo, W. A. van Gool, D. van de Beek, Systemic inflammation and microglial activation: systematic review of animal experiments. $J$ Neuroinflammation 12, 114 (2015).

48. S. Lehnardt, Innate immunity and neuroinflammation in the CNS: the role of microglia in Toll-like receptor-mediated neuronal injury. Glia 58, 253-263 (2010).

49. D. Erny, A. L. Hrabě de Angelis, M. Prinz, Communicating systems in the body: how microbiota and microglia cooperate. Immunology 150, 7-15 (2017).

50. F. Petrelli, L. Pucci, P. Bezzi, Astrocytes and Microglia and Their Potential Link with Autism Spectrum Disorders. Front Cell Neurosci 10, 21 (2016).

51. L. S. Abdelli, A. Samsam, S. A. Naser, Propionic Acid Induces Gliosis and Neuro-inflammation through Modulation of PTEN/AKT Pathway in Autism Spectrum Disorder. Scientific Reports 9, 8824 (2019).

52. J. Ellegood, J. N. Crawley, Behavioral and Neuroanatomical Phenotypes in Mouse Models of Autism. Neurotherapeutics 12, 521-533 (2015).

53. D. T. Stephenson et al., Histopathologic characterization of the BTBR mouse model of autistic-like behavior reveals selective changes in neurodevelopmental proteins and adult hippocampal neurogenesis. Mol Autism 2, 7 (2011).

54. Q. Zhang et al., Folic acid improves abnormal behavior via mitigation of oxidative stress, inflammation, and ferroptosis in the BTBR T+ tf/J mouse model of autism. J Nutr Biochem 71, 98109 (2019).

55. A. Nadeem et al., Increased oxidative stress in the cerebellum and peripheral immune cells leads to exaggerated autism-like repetitive behavior due to deficiency of antioxidant response in BTBR $\mathrm{T}+\mathrm{tf} / \mathrm{J}$ mice. Prog Neuropsychopharmacol Biol Psychiatry 89, 245-253 (2019).

56. J. Borovac, M. Bosch, K. Okamoto, Regulation of actin dynamics during structural plasticity of dendritic spines: Signaling messengers and actin-binding proteins. Mol Cell Neurosci 91, 122-130 (2018).

57. H. Takahashi, T. Mizui, T. Shirao, Down-regulation of drebrin A expression suppresses synaptic targeting of NMDA receptors in developing hippocampal neurones. $J$ Neurochem 97 Suppl 1, 110115 (2006). 
58. P. K. Yip et al., Galectin-3 released in response to traumatic brain injury acts as an alarmin orchestrating brain immune response and promoting neurodegeneration. Sci Rep 7, 41689 (2017).

59. P. Pesheva, S. Kuklinski, B. Schmitz, R. Probstmeier, Galectin-3 promotes neural cell adhesion and neurite growth. J Neurosci Res 54, 639-654 (1998).

60. L. P. Diniz et al., Astrocyte-induced synaptogenesis is mediated by transforming growth factor $\beta$ signaling through modulation of D-serine levels in cerebral cortex neurons. J Biol Chem 287, 4143241445 (2012).

\section{Figures}



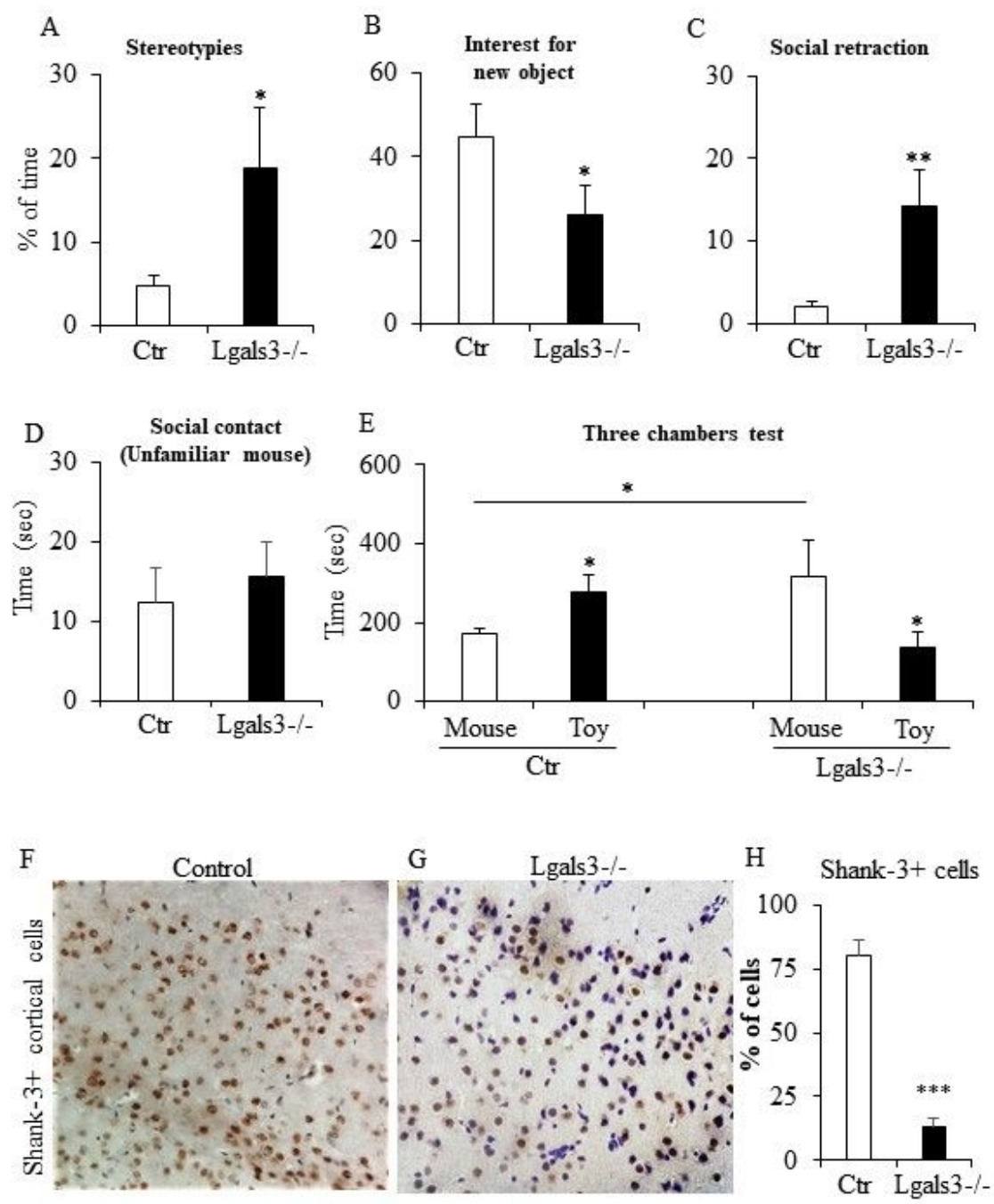

FIG 1

\section{Figure 1}

Behavioral tests and correlation with shank-3 expression in Lgals3-/- mice. (A) Stereotypies and repetitive motor patterns. (B) Interest for new object. (C) Social retraction. (D) Sociability with unfamiliar mouse. (E) Tree-Chambered test time spent exploring new toy and contact with same cage mice. Cells positive to Shank-3 (brown-stained cells) in control (F) and (G) Lgals3-/- mice. Data were obtained from three independent experiments. $\left.{ }^{*}\right) \mathrm{P} \leq 0.05$ and $\left({ }^{* *}\right) \mathrm{P} \leq 0.001$. 

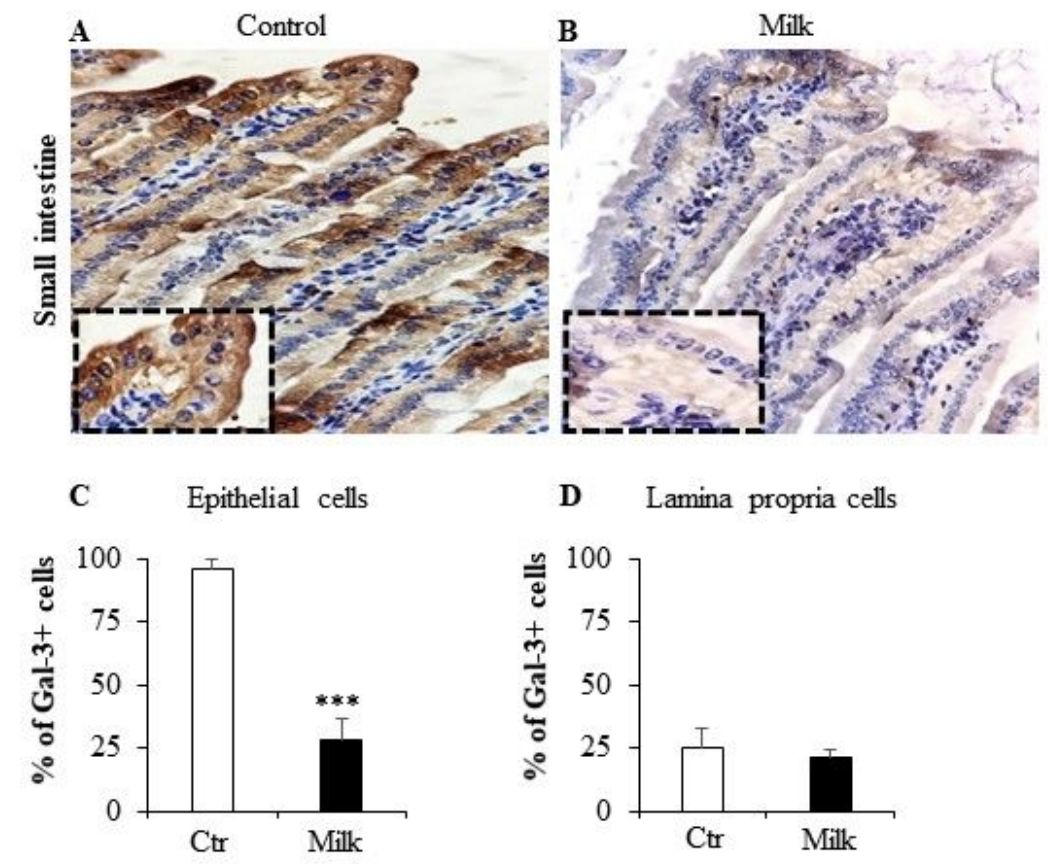

D Lamina propria cells

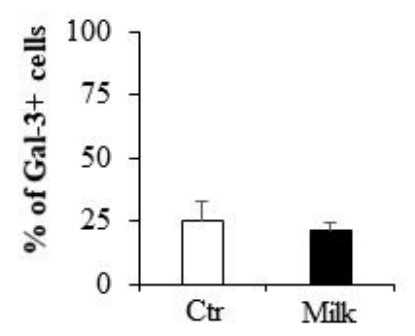

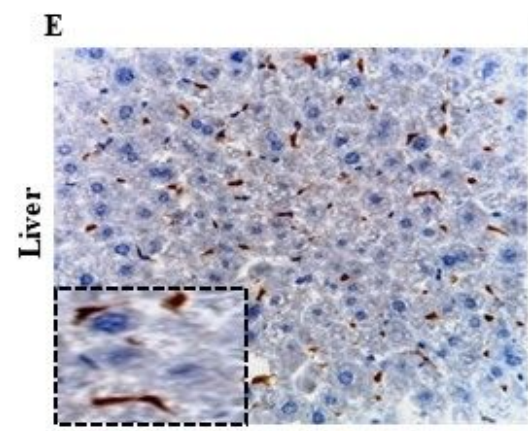

Control

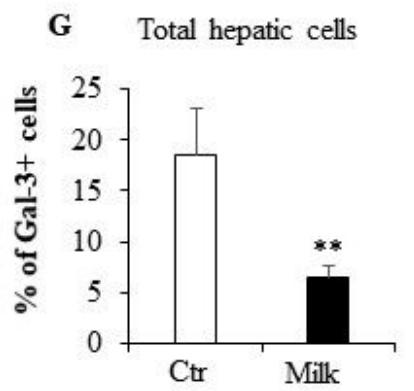

F

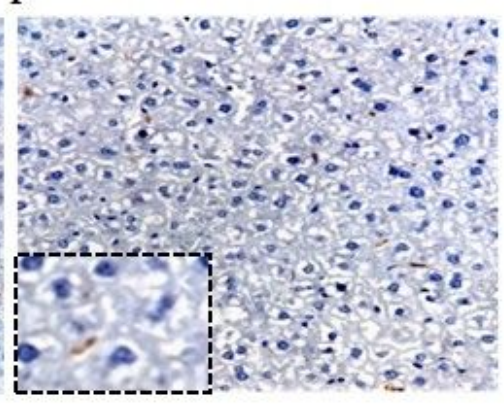

Milk

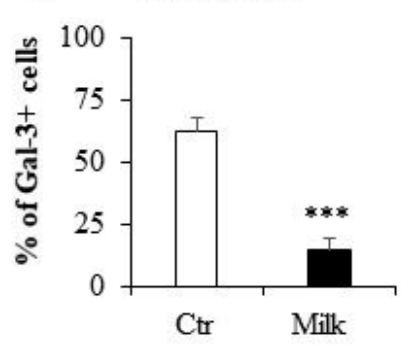

\section{FIG 2}

\section{Figure 2}

Intestinal and hepatic cells expressing galectin-3 in milk-supplemented mice. Enterocytes immunostained to galectin-3 in the gut of control mice (A) and after milk consumption for 7 days (B). Quantitative analysis of galectin-3+ cells in the intestinal epithelial cells (C) and lamina propria cells (D). Kupffer cells marked to galectin-3 in the liver of control mice (E) and after milk consumption for 7 days (F). Quantitative analysis of galectin-3+ cells in comparison with total hepatic cells (G) and compared with 
Kupffer cell population $(H)$. Data were obtained from three independent experiments. $\left.{ }^{* \star}\right) P \leq 0.01$ and $(\star \star \star) ~ P \leq 0.001$.
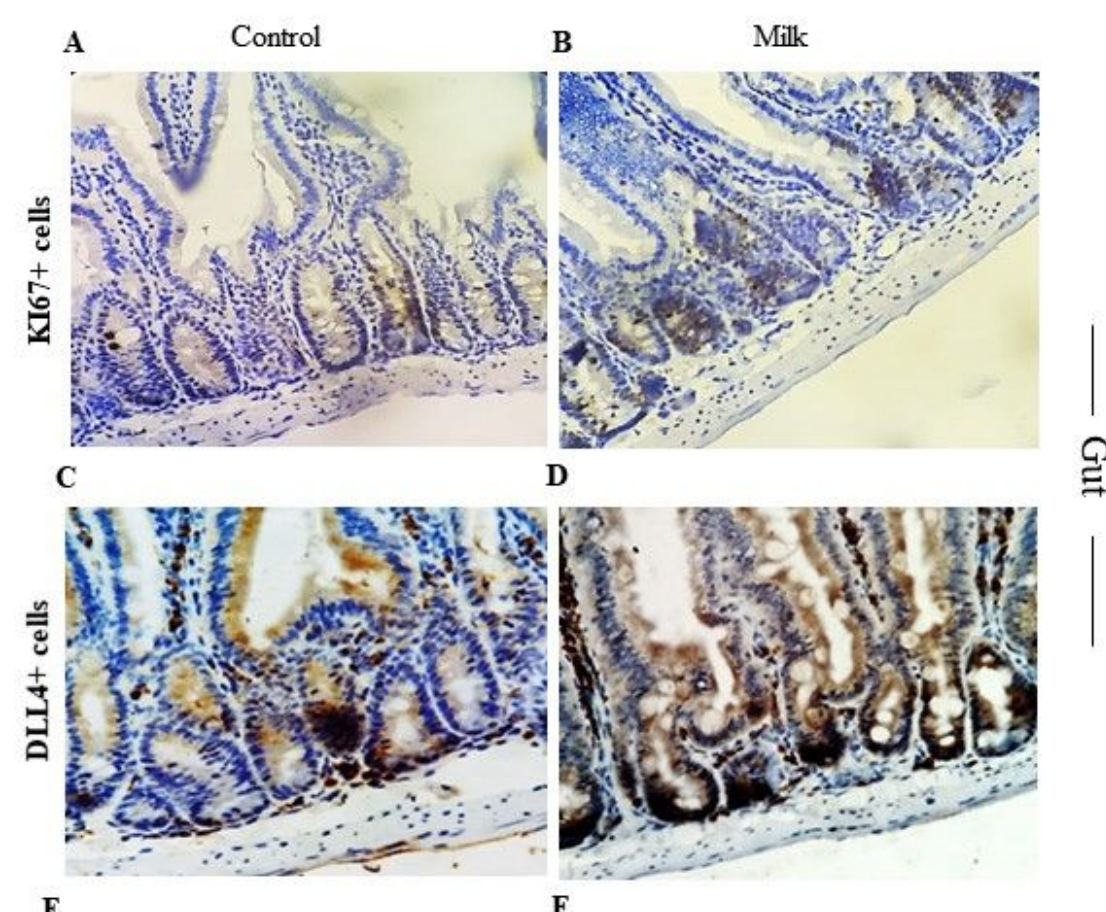

@

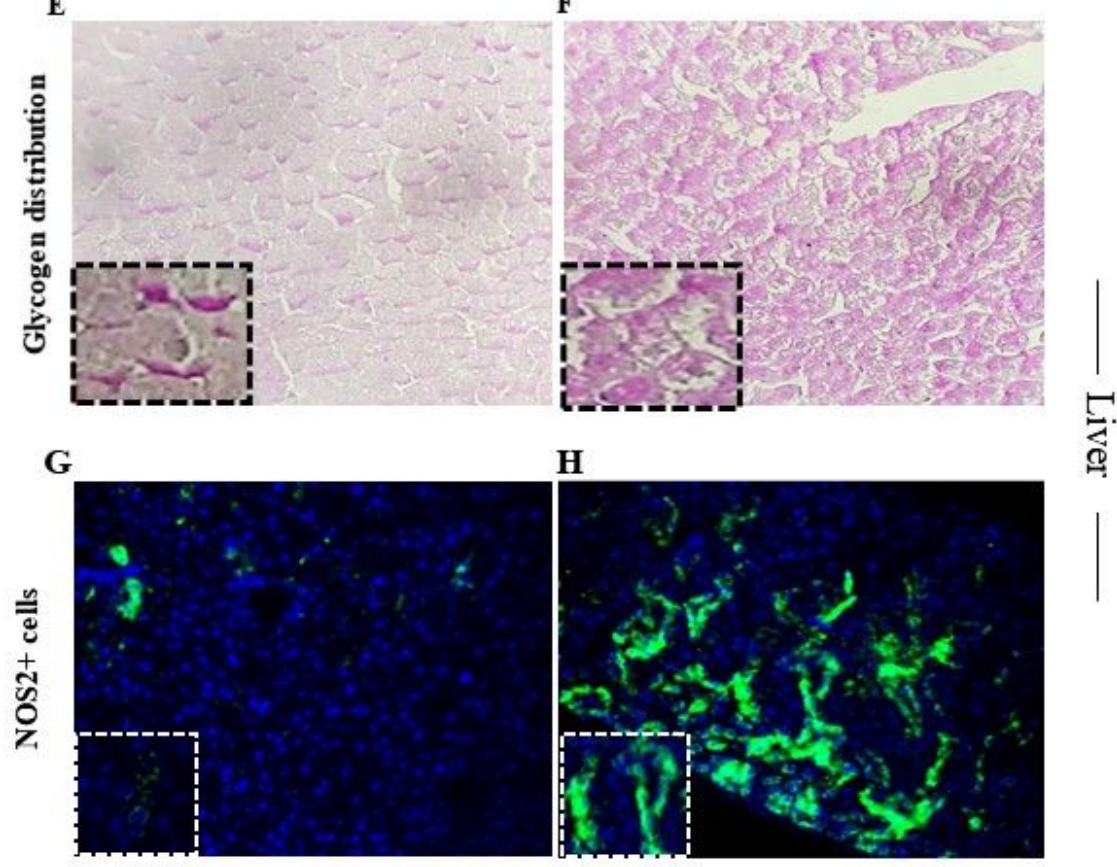

FIG 3

Figure 3

Histological disorders in the gut-liver axis caused by cow's milk consumption. Gut samples stained by immunohistochemistry to KI67 (A, controls; B milk-treated mice) and DLL-4 (C, controls; D, milk-treated mice). Liver samples were stained with PAS to observe glycogen distribution in controls (E) and milk- 
supplemented mice (F). Hepatic samples were also marked to NOS-2 and analyzed by fluorescence microscopy. Data were obtained from three independent experiments.
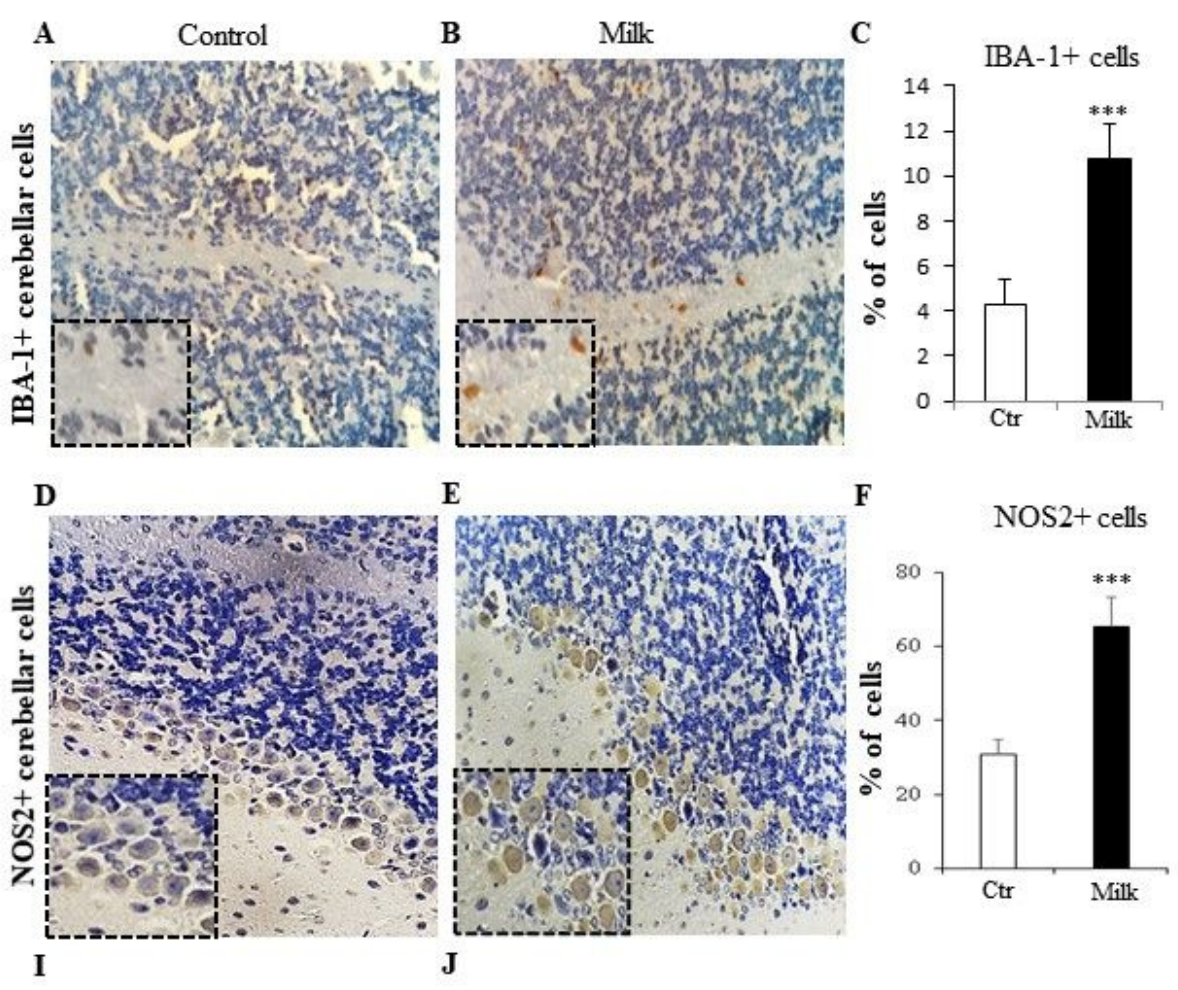

\section{FIG 4}

\section{Figure 4}

Photomicrographs of IBA-1 e NOS-2 in cerebellar cells after milk intake. Cerebellar Iba-1+ microglial cells were identified in control mice (A) and cow's milk supplemented mice (B) on day 7 of treatment. Bar graphs reveal the percentage of cerebellar Iba-1+ cells (C). NOS-2+ Purkinje cells were identified in the 
cerebellum of control mice (D) and cow's milk-supplemented mice (E) at the same time of treatment. Bar graphs reveal the percentage of cerebellar NOS-2+ cells (F). White bars represent controls while black bars represent milk-supplemented mice. Data were obtained from three independent experiments. (A, B, D and E) Magnification 400x. (**) $P \leq 0.01$.
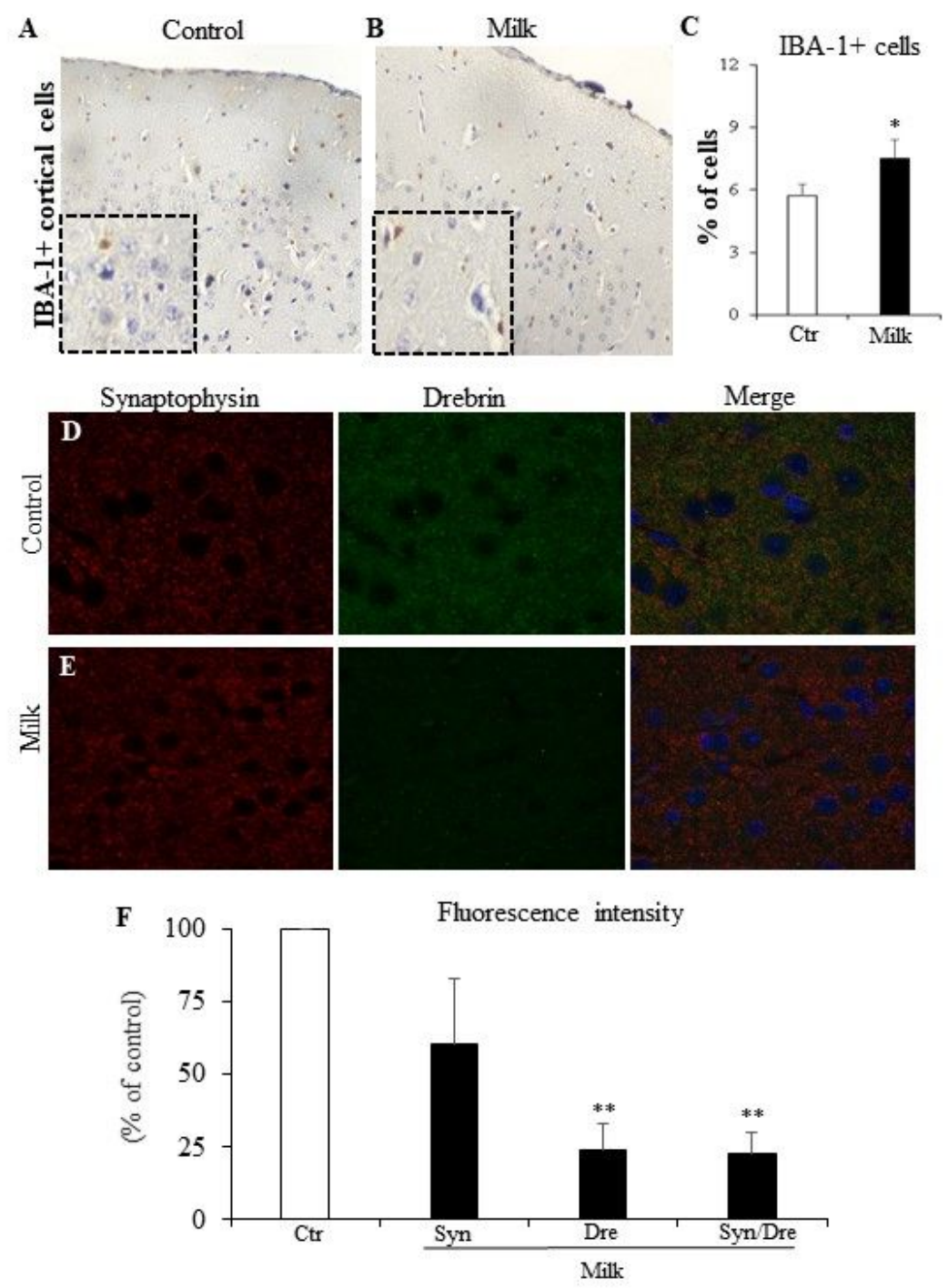

FIG 5

Figure 5 
Photomicrographs of cells expressing IBA-1 and synaptic proteins in cerebral cortex of mice after milk intake. Cells positive to Iba-1 were localized in cortico-cerebral regions of control (A) and mice supplemented with milk (B) on day 7 of treatment. Bar graphs indicate the percentage of cortico-cerebral Iba-1+ cells (C) in mice that received water (white bars) or milk (black bars). Cells positive to synaptophysin (green) and drebrin (red) were detected in control (D) and milk-supplemented mice (E). Bar graphs indicate the intensity of synaptophysin and drebrin in each group of mice (F). Data were obtained from three independent experiments. (A-B) Magnification 400x. (D-E) Magnification 200x. (*) $P \leq 0.05$ and $(\star \star) P \leq 0.01$. 

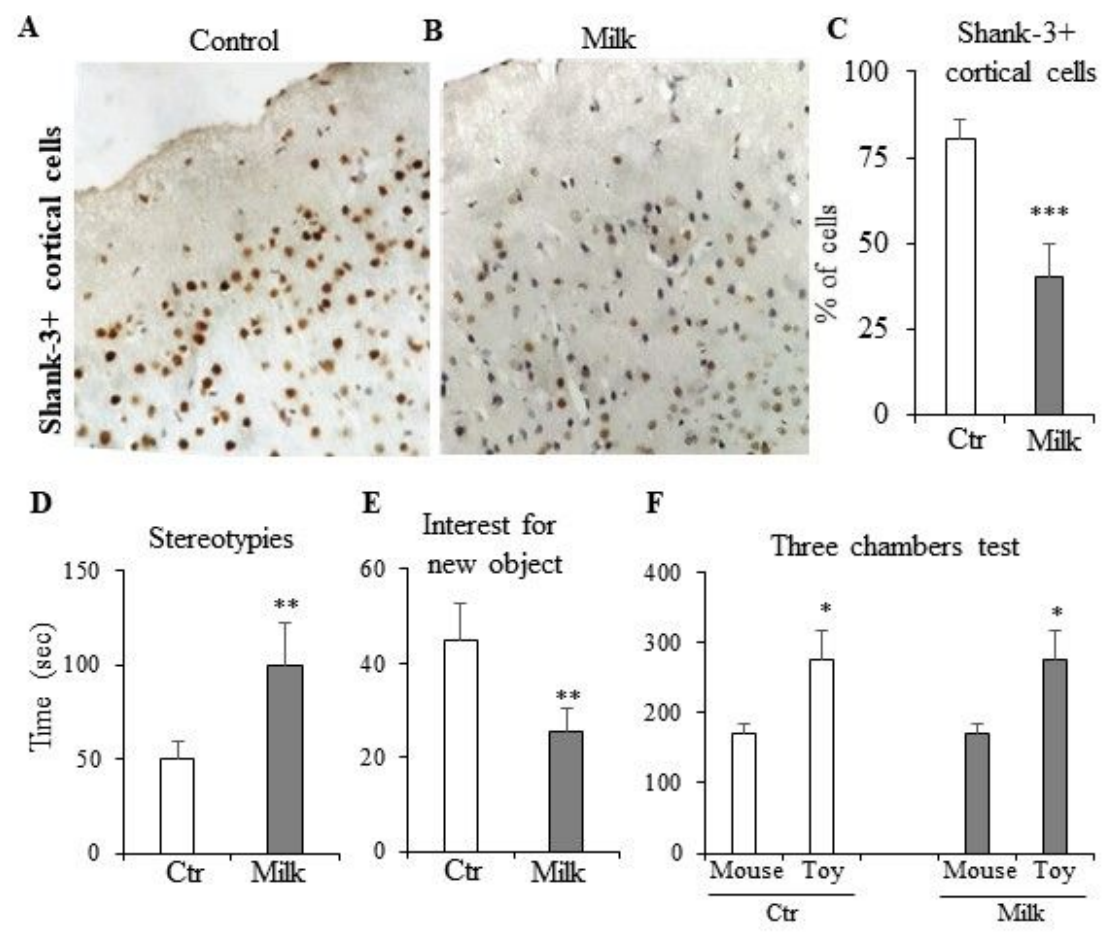

\section{FIG 6}

\section{Figure 6}

Photomicrographs of cortico-cerebral Shank-3 expressing cells and behavioral tests of milksupplemented mice. Cells positive to Shank-3 were identified in the cerebral cortex of control mice (A) and cow's milk-supplemented mice (B) on day 7 of treatment. Bar graphs reveal the percentage of cerebral Shank-3+ cells (C). Behavioral tests included (D) stereotypies and repetitive motor patterns, (E) interest for new object and $(F)$ tree-Chambered test time spent exploring new toy and contact with same cage mice 
Data were obtained from three independent experiments. (A-B) Magnification 400x. $\left.{ }^{\star}\right) P \leq 0.05$, $\left(^{\star *}\right)$ $P \leq 0.01$, and $(* \star *) P \leq 0.001$.

\section{Supplementary Files}

This is a list of supplementary files associated with this preprint. Click to download.

- Lemosetal2020SciRepSupplementaryfigures.docx 